\title{
Análise da influência do alcoolismo e tabagismo na fertilidade masculina
}

Analysis of the influence of alcoholism and smoking on male fertility

\author{
N. R. Gonçalves ${ }^{1 *} ;$ F. L. Dias ${ }^{1}$ \\ ${ }^{1}$ Laboratório de Citogenética e Mutagênese, Faculdades Integradas Aparício Carvalho-FIMCA e Metropolitana, Rua \\ Araras 241, Jardim Eldorado, CEP: 76912-640, Porto Velho-RO, Brasil
}

*naancontato@hotmail.com

(Recebido em 25 de fevereiro de 2016; aceito em 19 de maio de 2016)

\begin{abstract}
A infertilidade masculina é causada por fatores que bloqueiam ou alteram a espermatogênese. $\mathrm{O}$ alcoolismo e o fumo afetam a qualidade sexual e a capacidade de concepção no homem podendo desenvolver problemas de fertilidade. O objetivo deste estudo foi avaliar, através do exame Espermograma, se o alcoolismo e o tabagismo afetam a qualidade do sêmen. Foram analisados sêmens de 45 voluntários divididos em 03 grupos: 1. Grupo Controle, 2. Grupo Consumidor de Álcool e 3. Grupo Consumidor de Álcool e Fumo. Os resultados obtidos foram normais nas referências do teste, porém o Grupo Controle mostrou resultados com qualidade superior aos outros dois grupos. Na comparação de significância o Grupo Consumidor de Álcool teve a motilidade inferior ao Grupo Controle $(\mathrm{p}<0,05)$ e a morfologia, vitalidade, concentração espermática e concentração pelo volume ejaculado normais $(\mathrm{p}>0,05)$. Os resultados não apresentaram significância $(\mathrm{p}>0,05)$ entre o Grupo Controle e Grupo Consumidor de Álcool e fumo. Porém no Grupo Consumidor de Álcool e fumo foi obtido uma concentração espermática inferior ao Grupo Consumidor de Álcool $(\mathrm{p}<0,05)$. O exame revela informações importantes sobre as possíveis alterações que o álcool e o fumo podem causar nas características vitais do sêmen que são relevantes para homens susceptíveis a infertilidade.

Palavras-chave: infertilidade, alcoolismo, tabaco.
\end{abstract}

Male infertility is caused by factors that block or alter spermatogenesis. Alcoholism and smoking affect sexual quality and conception capability in men and may cause the development of fertility problems. The objective of this study was to evaluate, through a semen examination whether or not alcoholism and smoking affect semen quality. Semen from 45 volunteers divided into 03 groups were analyzed: 1 . Control Group 2. Alcohol Consumer Group and 3. Alcohol and Tobacco Consumer Group. The test results were normal, but the control group showed results superior to the other two groups. In a significance comparison the Alcohol Consumer Group had an motility lower than the control group $(\mathrm{p}<0.05)$ and normal motility, morphology, vitality, spermatic concentration and concentration by volume ejaculated ( $p>0.05$ ). Results were not significant ( $p>0.05$ ) between the Control Group and Alcohol and tobacco Consumer Group. But the Alcohol and tobacco Consumer Group obtained a lower spermatic concentration than the Alcohol Consumer Group ( $\mathrm{p}$ < 0.05). The test reveals important information about the possible alterations that alcohol and tobacco can have on vital semen characteristics that are relevant to men susceptible to infertility.

Keywords: infertility, alcoholism, tobacco.

\section{INTRODUÇÃO}

O consumo de álcool no Brasil tem sido crescente entre jovens de 12 a 24 anos a uma proporção de consumo igual entre os sexos. Na nossa sociedade a iniciação do consumo destas bebidas, sobretudo a cerveja, é idealizada como um processo de transformação do jovem em adulto [1, 2, 3, 4]. O álcool e o cigarro aparecem em quase 90\% das opções de lazer dos jovens, nas baladas, como símbolo de beleza, juventude, sexualidade, força e sucesso $[1,4,5]$.

Os estudos comprovam que o consumo de derivados do tabaco causa cerca de 50 doenças diferentes, principalmente doenças cardiovasculares, respiratórias, câncer e impotência sexual no homem $[2,6,7]$. O álcool, apesar de aparentemente ser menos propício a causar danos, seus 
efeitos são tão nocivos para a fertilidade masculina quanto os do fumo e ainda pode intensificar os efeitos quando associado a uma doença $[2,7,8]$.

Durante o ato sexual, a qualidade do desempenho dos fumantes é pior do que a de indivíduos não fumantes, e também menor a capacidade de concepção $[9,10]$. Pois, a nicotina atinge diretamente as células produtoras de espermatozóides nos fumantes, aumentando o número de formas imaturas, sobretudo em homens acima de 30 anos $[6,8,11,12]$. Já o álcool, em excesso pode trazer problemas semelhantes para qualquer idade, diminui o desempenho e o desejo sexual, atrofia as células produtoras de testosterona, interfere na irrigação sanguínea ligada a ereção e diminui o número e a saúde física dos espermatozoides [7, 8].

Estima-se que até $15 \%$ dos casais no mundo apresentam algum problema de fertilidade, sendo $40 \%$ por problemas masculinos $[13,14,15,16]$. Para que ocorra corretamente o processo da fecundação e fertilização entre o espermatozóide e o óvulo é necessário que um conjunto de parâmetros esteja fisiologicamente normal $[13,14]$. Dentre os mais importantes temos o hormônio testosterona, vesículas seminais, próstata, testículos e outros [9, 10]. Considera-se infertilidade quando não ocorre o processo de gravidez em torno de um ano de tentativas sem utilização de interferentes $[8,17]$.

O espermograma é um exame objetivo e eficiente que avalia os parâmetros de qualidade do sêmen para uma avaliação diagnóstica do estado fértil masculino [4, 18, 19]. O formato do espermatozóide é uma das informações mais importante para se determinar a qualidade do sêmen. O espermatozóide normal tem cabeça oval, a peça intermediária e flagelo normais $[9,14,20]$; já o do fumante pode apresentar formato fusiforme, de pêra ou amorfos, parte intermediária e flagelo defeituosos que dificultam a fecundação $[6,12,21]$.

Este trabalho foi desenvolvido com homens voluntários integrantes do ambiente acadêmico das Faculdades FIMCA e METROPOLITANA da cidade de Porto Velho-RO, com o objetivo de avaliar se o tabagismo e o alcoolismo influenciam e/ou causam alterações na qualidade do esperma. Para isso, os resultados do espermograma dos grupos consumidores de álcool e grupos consumidores de álcool e tabaco foram comparados com o grupo controle (formado por indivíduos que não consomem álcool e tabaco).

\section{MATERIAL E MÉTODOS}

O presente trabalho foi realizado no Laboratório de Citogenética e Mutagênese das Faculdades Integradas Aparício Carvalho-FIMCA e METROPOLITANA, no período de Agosto de 2011 a Junho de 2012 e contou com a aprovação do comitê de ética da instituição: Carta 66/2011, CAAE-0008.0.382.000-11. Após a concordância e assinatura do termo de consentimento livre e esclarecido foi colhido o líquido seminal de 45 voluntários do ambiente acadêmico dessas Faculdades (alunos, funcionários e visitantes) com idades entre 22 a 38 anos, divididos em 3 grupos: Grupo Controle (GC) - composto por 15 indivíduos que não fumam, não consomem bebidas alcoólicas e sem outra exposição de risco; Grupo Consumidor de Álcool (GA) composto por 15 indivíduos consumidores de bebidas alcoólicas e não fumantes, sem outros fatores de riscos na avaliação; e Grupo Consumidor de Álcool e Fumo (GAF) - composto por 15 indivíduos consumidores de bebidas alcoólicas e fumantes, sem exposição a outros fatores de risco. As informações sobre os fatores de riscos considerados foram obtidas por um questionário feito previamente com perguntas sobre idade, sexo, tabagismo, alcoolismo, histórico de saúde, problemas genéticos e exposição a radiação, consumo de medicamentos ou outros químicos que poderiam interferir farmacologicamente nos resultados. Nem um dos indivíduos da pesquisa apresentavam a princípio quadro de infertilidade diagnosticado, sendo assim os resultados da pesquisa foram avaliados pelas referências do teste e pela comparação com o Grupo Controle.

No procedimento, o Espermograma [22, 23, 24, 25] foi realizado duas vezes em cada indivíduo com intervalos de duas a oito semanas do primeiro para o segundo, respeitando o período de 3 a 5 das de abstinência. A média dos dois exames foi obtida e analisada, entrando em pauta uma terceira amostra se necessária, em caso de divergência dos resultados. $\mathrm{O}$ exame foi dividido em duas análises: Análise Macroscópica e Análise Microscópica. As amostras foram coletadas por masturbação dentro de recipiente plástico estéril e após intervalo de 30 minutos, foram iniciadas as análises. Depois da avaliação macroscópica dos parâmetros cor (CR), odor 
(OD), aspecto (AS) e liquefação (LQ) as amostras foram aspiradas com o auxílio de uma pipeta graduada acoplada a um pipetador eletrônico para determinação do volume (VL) e viscosidade (VS). Para realização das análises microscópicas o conteúdo seminal foi cuidadosamente homogeneizado e uma alíquota de $10 \mu \mathrm{L}$ foi utilizada para confeccionar esfregaço a fresco na determinação da motilidade (MT) dos espermatozóides, bem como $10 \mu \mathrm{L}$ para confecção de esfregados corados com corante Giemsa e outro com Panótipo para avaliação da morfologia (MF) e vitalidade (VT), respectivamente. Uma alíquota de $10 \mu \mathrm{L}$ foi inserida em uma câmara de Neubauer $(0,01 \mu \mathrm{L}$ de volume) para determinação das concentrações espermáticas (CE) (n x $10^{6} / \mathrm{mL}$ ) e volume ejaculado (CV) (n x 10\%/volume ejaculado). As análises microscópicas foram realizadas com o uso de microscópio óptico com objetiva de aumento 40x e/ou 100x.

Os resultados da análise macroscópica foram comparados pelas características e em porcentagens obtidas entre os grupos. O teste $t$ de Student foi utilizado para comparar as médias dos resultados examinados entre os grupos na análise microscópica. O nível de diferença estatística estabelecido foi $5 \%$.

\section{RESULTADOS}

As informações coletadas nos questionários (Tabela 1) mostram que os três grupos apresentam um mesmo perfil de faixa etária, são compostos por solteiros e casados, cumpriram um tempo de abstinência de acordo com o exigido pelo exame, não existiu relato de caso determinante no histórico de saúde, caso familiar, casos de doença genética, riscos de exposição à radiação ou outros fatores de riscos determinantes. A média do consumo de álcool pelo GA foi cerca de 2800 $\mathrm{mL}$ por semana em um tempo médio de 7 anos. No GAF a média do consumo de bebida e cigarro foi de $2500 \mathrm{~mL}$ por semana em 9 anos e 8 cigarros por dia em 8,5 anos, respectivamente.

Tabela 1: Dados obtidos dos voluntários por meio do questionário.

\begin{tabular}{|c|c|c|c|}
\hline & Grupo Controle & $\begin{array}{c}\text { Grupo Consumidor de } \\
\text { Álcool }\end{array}$ & $\begin{array}{l}\text { Grupo Consumidor } \\
\text { de Álcool e Fumo }\end{array}$ \\
\hline Faixa etária & $>22$ anos $a<38$ anos & $>22$ anos a =38anos & $>22$ anos $\mathrm{a}=38 \mathrm{anos}$ \\
\hline Estado civil & $11 \mathrm{sol} / 4 \mathrm{cas}$ & $9 \mathrm{sol} / 6 \mathrm{cas}$ & $10 \mathrm{sol} / 5 \mathrm{cas}$ \\
\hline Tempo de abstinência & 03 a 04 dias & 03 a 04 dias & 03 a 04dias \\
\hline Consumo de álcool & Não & *2800 mL/semana & **2500 mL/semana \\
\hline Consumo de Tabaco & Não & Não & $* * * 8$ cigarros/dia \\
\hline Histórico de saúde & Não & Não & Não \\
\hline Histórico genético & Não & Não & Não \\
\hline Exposição a radiação & Não & Não & Não \\
\hline Fatores de risco & Não & Não & Não \\
\hline
\end{tabular}

*Tempo médio de 7 anos; **Tempo médio de 9 anos; ***Tempo médio de 8,5 anos. sol=solteiro; cas $=$ casado

Os resultados apresentados fazem uma comparação das características analisadas de cada grupo pelos padrões do exame. A avaliação da qualidade das amostras na análise macroscópica levando em consideração os resultados entre os 3 grupos obteve 15 resultados com coloração branco-opaco e nenhum amarelo-claro para o GC e GA, mas para o GAF foram 12 resultados branco-opaco e 3 amarelo-claro. O OD foi característico (Sui generis) nas análises dos 3 grupos, com exceção de 1 resultado descaracterístico apresentado no GAF. Em geral, observou-se o AS fluido para o GC, no entanto, a quantidade de AS levemente alterado foi de 2 e 4 para o GA e GAF, respectivamente. O GC teve 1 resultado de VS levemente alterado, enquanto que o GA obteve 2 e o GAF 4 resultados levemente alterados. A medida do VL das amostras do GC e GA apresentaram 1 um resultado com volume inferior a $2 \mathrm{~mL}(\leq 2 \mathrm{~mL})$, os demais medindo de 2 a 5 $\mathrm{mL}$ ( $\geq 2 \mathrm{a} \leq 5 \mathrm{~mL}$ ), e 2 resultados $\leq 2 \mathrm{~mL}$ para o GAF. O tempo decorrido para total liquefação (LQ) das amostras foi diferente entre os grupos, onde o GC apresentou média (MD) $=35,8$ minutos (Desvio Padrão - $\mathrm{DP}=0,9)$, o GA $\mathrm{MD}=39,1$ minutos $(\mathrm{DP}=1,3)$ e o $\mathrm{GAF} 38,4$ minutos 
$(\mathrm{DP}=2,1)$. A MD da aferição do $\mathrm{pH}$ entre os grupo foi de 7,5 para o $\mathrm{GC}(\mathrm{DP}=0,2), 7,7(\mathrm{DP}=0,1)$ para o GA e 7,8 $(\mathrm{DP}=0,4)$ para o GAF. Estes dados podem ser observados na tabela 2.

Tabela 2: Comparação dos resultados da análise macroscópica do Espermograma entre os grupos Controle, Consumidor de Álcool e Consumidor de Álcool e Fumo

G R U P O S

\begin{tabular}{|c|c|c|c|c|c|}
\hline & & & Grupo Controle & $\begin{array}{c}\text { Grupo Consumidor } \\
\text { de Álcool }\end{array}$ & $\begin{array}{c}\text { Grupo Consumidor } \\
\text { de Álcool e Fumo }\end{array}$ \\
\hline \multirow{14}{*}{ 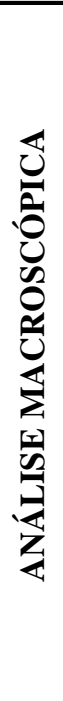 } & \multirow{2}{*}{ Cor } & Branco-opaco & 15 & 15 & 12 \\
\hline & & Amarelo-claro & -- & - & 3 \\
\hline & \multirow{2}{*}{ Odor } & Sui Generis & 15 & 15 & 14 \\
\hline & & Descaracterístico & -- & -- & 1 \\
\hline & \multirow{2}{*}{ Aspecto } & Fluido & 15 & 13 & 11 \\
\hline & & Levemente Alterado & -- & 2 & 4 \\
\hline & \multirow{2}{*}{ Volume } & $\geq 2 \mathrm{a} \leq 5 \mathrm{~mL}$ & 14 & 14 & 13 \\
\hline & & $\leq 2 \mathrm{~mL}$ & 1 & 1 & 2 \\
\hline & \multirow{2}{*}{ Liquefação } & $\leq 50 \mathrm{~min}$ & 15 & 15 & 15 \\
\hline & & $\geq 50 \mathrm{~min}$ & -- & -- & -- \\
\hline & \multirow{2}{*}{ Viscosidade } & Normal & 14 & 13 & 11 \\
\hline & & Levemente Alterado & 1 & 2 & 4 \\
\hline & \multirow{2}{*}{ pH } & $7-8$ & 15 & 11 & 10 \\
\hline & & $8-9$ & -- & 4 & 5 \\
\hline
\end{tabular}

A análise microscópica dos parâmetros de MT, VT, MF, CE e CV apresentou os seguintes resultados (conforme Figura 1): a MT dos espermatozoides teve MD de 88,5\% (DP=3,6) no GC, $82,3 \%(\mathrm{DP}=5,4)$ no GA e 79,2\% ( $\mathrm{DP}=7,0)$ no GAF. Na comparação da quantidade de células vivas e mortas (VT) obteve-se MD de 88,9\% (DP=1,5) no GC, $81,5 \%(\mathrm{DP}=3,8)$ no GA e $80,5 \%$ $(\mathrm{DP}=4,4)$ no GAF. A avaliação da $\mathrm{MF}$ no $\mathrm{GC}$ apresentou $\mathrm{MD}=83,9 \%(\mathrm{DP}=4,4), \mathrm{GA} \mathrm{MD}=77,1 \%$ $(\mathrm{DP}=4,0)$ e $\mathrm{GAF} \mathrm{MD}=75,3 \%(\mathrm{DP}=5,1)$. A contagem do número de células por $\mathrm{mL}(\mathrm{CE})$ no $\mathrm{GC}$ a MD obtida foi de $164,8 \times 10^{6} / \mathrm{mL}(\mathrm{DP}=16,3)$, no GA $123,2 \times 10^{6} / \mathrm{mL}(\mathrm{DP}=10,8)$ e no GAF $116,7 \times 10^{6} / \mathrm{mL}(\mathrm{DP}=11,5)$. E por fim, a comparação do número de espermatozoides pela $\mathrm{CV}$ do GC teve MD de 295,8 x10\%/volume ejaculado (DP=19,7), o GA 265,2 x10\% volume ejaculado $(\mathrm{DP}=13,4)$ e GAF $277,4 \times 10^{6} /$ volume ejaculado $(\mathrm{DP}=16,2)$ (Figura 1$)$.
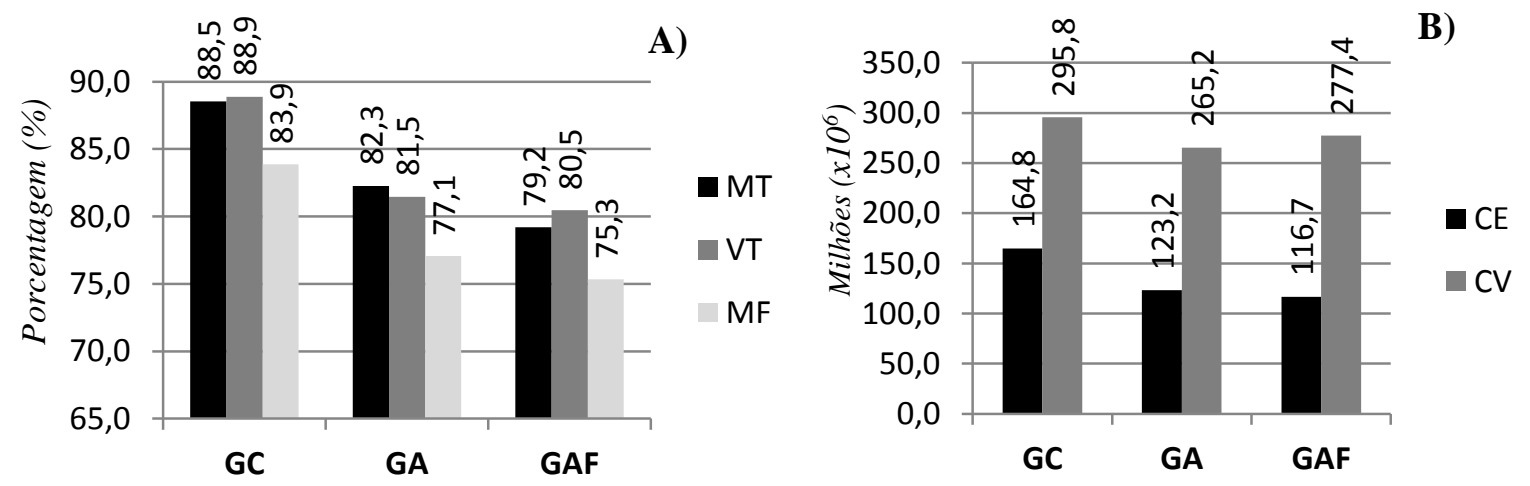

Figura 1: Comparação dos resultados da análise microscópica entre os grupos GC, GA e GAF. Na parte A da figura estão apresentadas as médias percentuais de MT, VT e MF. E na parte B as médias dos valores da CE e CV. GC = Grupo Controle; GA = Grupo Alcoólico; GAF = Grupo Alcoólico e Fumante; $M T=$ Motilidade; $V T=$ Vitalidade $; M F=$ Morfologia $; C E=$ Concentração Espermática $\left(n \times 10^{6} / \mathrm{mL}\right) ; C V=$ Concentração pelo volume ejaculado ( $\mathrm{n} \times 10^{6}$ /volume ejaculado).

No que se refere à significância foi observado na comparação da análise microscópica que houve um resultado da MT dos espermatozóides significativamente menor do GA $(p<0,05)$ em 
relação ao GC, e as demais VT, MF, CE e CV ficaram normais ( $p>0,05$ ). Na comparação entre o GC e GAF todos os resultados (MT, VT, MF, CE e CV) não apresentaram significância ( $p$ > $0,05)$. Os resultados de MT, VT, MF e CV não tiveram valor de significância $(p>0,05)$ entre o GA e GAF, mas deste último a $\mathrm{CE}$ apresentou um número de células menor $(\mathrm{p}<0,05)$ em comparação ao GA.

\section{DISCUSSÃO}

A ocorrência de infertilidade é fruto de um desenvolvimento multifatorial que pode ser diagnosticado logo na juventude, ou em idade mais avançada. Às vezes, ocorre gradativamente e afeta até mesmo homens que já tenham sido pais anteriormente [19, 26, 17]. Outra questão se detém ao tempo e as drogas como álcool e fumo que não são consideradas agentes perigosos à fertilidade, sobretudo pelos jovens, porque as consequências advindas são a longo prazo $[2,4]$.

A principal forma de avaliar as condições fisiológicas do conteúdo espermal é pelo espermograma. Numa análise quantitativa e qualitativa são verificadas características físicas e químicas se obtendo resultados diagnósticos importantes sobre a saúde dos espermatozóides. Entender as alterações nos resultados é importante para identificação do agente ou causa responsável pelo problema incidente [19, 27, 28].

Neste estudo, os voluntários dos três grupos não apresentaram resultados alterados ou essas alterações foram pouco expressivas em termos diagnósticos, sendo normais nos intervalos de referências do teste. Os dados informados pelos voluntários dos três grupos mostram que todos dispõem de boa saúde, sem possível suscetibilidade de alterações físicas ou químicas (Tabela 1). A média de exposição ao álcool e fumo se faz determinante na avaliação, visto que podem causar alterações quando o consumo, tanto de álcool como do cigarro, é feito de forma intensa e por longo prazo [6, 29]. Esse tipo de avaliação do histórico clínico dos pacientes deve preceder as etapas analíticas, pois constitui um conjunto de informações importantes na elucidação da infertilidade masculina, como descrevem alguns autores em suas publicações [13, 14, 30, 31].

Aghamohammadi e Zafari (2011) [32] analisaram as consequências do tabagismo nos parâmetros de avaliação no esperma de 180 parceiros de casais inférteis em Sari no Irã. As amostras foram analisadas e comparadas entre homens fumantes $(21,7 \%)$ e não fumantes $(78,3 \%)$. Dos pacientes analisados, os resultados dos parâmetros avaliados não tiveram nenhuma diferença significativa na comparação entre os grupos.

Nos resultados obtidos neste trabalho houve uma diminuição significativa da motilidade dos espermatozóides do grupo GA ( $\mathrm{p}<0,05$ ) em comparação ao grupo GC, e também do número de espermatozoides contados por $\mathrm{mL}(\mathrm{CE})$ do $\operatorname{GAF}(\mathrm{p}<0,05)$ em relação ao GA (Figura 1). A motilidade é importante para que os espermatozóides alcancem o óvulo, para isso eles devem estar ativos, com movimentos rápidos e progressivos, em conciliação com o número suficiente de células na contagem de espermatozoides para aumentar as chances da fertilização. As causas de diminuição de espermatozoides podem decorrer de pouca abstinência sexual, por infecções ou distúrbios genéticos $[13,8,20]$. E entre os agentes causadores de alterações na qualidade do sêmen, reduzindo a concentração e motilidade das células estão o álcool e o fumo [12].

Os resultados comparados são resultantes da média dos valores obtidos da primeira e segunda análise do espermograma que em conjunto formam esse parâmetro de avaliação entre os grupos (Tabela 2 e Figura 1 A, B). Embora que as normalidades de todos os resultados foram satisfatórias, a avaliação comparativa mostra que o GC apresenta, quase como um todo, resultados com melhor qualidade, sobretudo em relação ao GAF. Considerando que a qualidade é importante para determinação de qualquer resultado analisado, isto implica que, melhor resultado indica melhor qualidade da amostra $[6,12,21,32]$.

Ahmadnia e colaboradores (2007) [33] fizeram um estudo de avaliação da espermatogênese de ratos expostos à fumaça do cigarro, divididos em dois grupos, teste e um controle, num total de 30 ratos. Os ratos do grupo teste foram expostos à fumaça do cigarro enquanto que o grupo controle foi mantido em ambiente fresco. Observaram-se resultados significantes na redução de desenvolvimento dos espermatozóides para os dois grupos, no entanto o grupo teste apresentou alterações determinantes no diâmetro dos túbulos seminíferos, no número de células de Sertoli, germinativas e Leydig. 
Um estudo de determinação dos efeitos do cigarro ou cachimbo de água nos níveis séricos dos hormônios LH, FSH e testosterona em ratos machos mostraram que os resultados foram insignificantes nos níveis sérios de LH e FSH para os ratos fumadores de cachimbo de água, mas foram significantes para os que fizeram uso do cigarro, em comparação aos controles. Os níveis séricos de testosterona reduziram tanto nos ratos expostos ao cachimbo de água como ao cigarro [34].

Os resultados do presente estudo não avaliam um possível diagnóstico de fertilidade masculina, porém oferece informações importantes relativas à quantidade e qualidade dos espermatozóides que condizem com os estudos usados no referencial. No entanto, o exame mostra as possíveis influências do álcool individual ou em conjunto com o cigarro que podem causar no líquido seminal, pelos termos qualitativos e quantitativos apresentados nos resultados dos três grupos [7,33].

Além do Espermograma, outros exames devem ser realizados na avaliação do sêmen em um diagnóstico de infertilidade. Diversos testes podem ser feitos para investigação das possíveis alterações envolvidas no caso, como testes funcionais dos espermatozóides, dosagens dos hormonais, exame histopatológico dos testículos, análise cariótipa [18, 19, 27, 35], busca de anticorpos, ultrassonografia escrotal e teste de fragmentação do espermatozoide [36], para ser somados aos resultados do espermograma e desfeche do diagnostico.

\section{CONCLUSÃO}

Os resultados das amostras dos voluntários analisadas neste trabalho apresentaram dados normais. Entretanto, observa-se uma discreta significância de menor MT no GA ( $p<0,05)$ em comparação ao GC e uma CE mais baixa do GAF $(p<0,05)$ em relação ao GA. Portanto, aos indivíduos que apresentam baixa qualidade seminal ou histórico pertinente de infertilidade, o contato com álcool e fumo pode induzir um agravo clínico. Nestes casos, a abstinência é o meio mais seguro para se preservar e evitar a tempo um futuro caso de infertilidade.

\section{REFERÊNCIAS BIBLIOGRÁFICAS}

1. BRASIL (A). Secretaria Nacional de Políticas sobre Drogas. Drogas: Cartilha álcool e jovens. Adaptação para 2. ed. Brasília; 2010. 44 p.

2. BRASIL (B). Secretaria Nacional de Políticas sobre Drogas. I Levantamento Nacional Sobre o Uso de Álcool, Tabaco e Outras Drogas entre Universitários das 27 Capitais Brasileiras. GREA/IPQHC/FMUSP. Brasília: SENAD; 2010. 284 p.

3. Fonseca MS. Aquisição de drogas: um estudo entre estudantes brasileiros. Psico-USF. 2002 Jul/Dez; 7(2): 153-162.

4. Vier BP, Rego-Filho EA, Campos E, et al. Uso de álcool e tabaco em adolescentes. Arq. Mudi. 2007 Ago; 11(2): 5-8.

5. Abramovay M, Castro MG. Drogas nas escolas. Versão resumida/Miriam Abramovay, Mary Garcia Castro. UNESCO, Rede Pitágoras. Brasília; 2005. 143 p.

6. Oliveira ÁAV. Efeito do Consumo do Tabaco na Fertilidade Masculina [dissertação]. Covilhã (PT): Faculdade de Ciências da Saúde, Universidade da Beira Interior; 2010. 57 p.

7. Sassine VJ. Álcool e fumo afetam fertilidade. Jornal O Popular [internet]. 2010 fev. Disponível em: http://www2.ucg.br/flash/Flash2010/Fevereiro10/100208repro.html.

8. Barbosa FFS. Influência dos antioxidantes na qualidade do sêmen de homens em tratamento de fertilidade [dissertação]. Lisboa (PT): Faculdade de Ciências, Universidade de Lisboa; 2009. 70 p.

9. Carvalho OF, Ferreira JDJ, Silveira NA, et al. Efeito oxidativo do óxido nítrico e infertilidade no macho. JBPML. 2002 Jul ; 38(1): 33-38, dx.doi.org/10.1590/S1676-24442002000100007.

10. Silva LFI, Petersen CG, Oliveira JBA, et al. Idade masculina: qualidade do sêmen e fertilidade. JBRA Reprod Assist. 2012 Mai/Jun; 16(3): 91-95.

11. Mostafa T. Cigarette smoking and male infertility. Journal of Advanced Research [internet]. $2010 \mathrm{Jul}$; 1(3): 179-186, doi:10.1016/j.jare.2010.05.002.

12. Lima VM, Barbieri RL, Aquila AD, et al. Estudo histopatológico do efeito da nicotina nos testículos e epidídimos de ratos. Rev Bras Clin Med [internet]. 2012 Jul/Ago; 10(4): 322-328.

13. Gonçalves J. Avaliação do casal infértil. Rev Port Clin Geral [internet]. 2005 Set/Out; 21(5): 493-503. 
14. Rosenblatt C, Filho MAD, Delgado DR, et al. Infertilidade Masculina - novos conceitos. Prática Hospitalar [internet]. 2010 Set/Out; Ano XII (71): 85-92.

15. Pasqualotto FF. Investigação e reprodução assistida no tratamento da infertilidade masculina. Rev Bras Ginecol Obstet. 2007 Fev; 29(2): 103-112, dx.doi.org/10.1590/S0100-72032007000200008.

16. Terraciano PB, Miquelito LV, Baptista LP, et al. Análise das alterações espermáticas e dos índices de fertilização de pacientes submetidos à reprodução assistida. Revista HCPA. 2006 Set; 26(1): 125.

17. Santos TRM, Santos DN, Barreto CS, et al. Considerações Sobre Infertilidade Masculina. Cadernos de Graduação - Ciências Biológicas e da Saúde [internet]. 2013 Mar; 1(16): 21-26.

18. Silva LF, Carvalho F. Actualizações na infertilidade masculina. Edição Sociedade Portuguesa de Andrologia. SAÚDE, SÁ. 2011; 311030/10. 115 p. Disponível em: http://docplayer.com.br/3054944Actualizacoes-na-infertilidade-masculina.html.

19. Kussler AP, Coitinho AS. Técnicas de reprodução assistida no tratamento da infertilidade. RBAC. 2008; 40(4): 313-315.

20. Pasqualotto FF, Fonseca GP, Silva ML, et al. Influência do período de abstinência sexual sobre as características seminais de homens inférteis. Rev Bras Ginecol Obstet [internet]. 2006 Jan; 28(1): 44 49, dx.doi.org/10.1590/S0100-72032006000100008.

21. Bouvet BR, Paparella CV, Feldman RN. Effect of tobacco consumption on the spermatogenesis in males with idiopathic infertility. Archivos Espanoles de Urologia. 2007; 60(3): 273-277. PMID: 17601302.

22. Strasinger SK, Di Lorenzo MS. Urinálise e Fluidos Corporais. 5. ed. São Paulo: Livraria Medica Paulista; 2009. capítulo 11, sêmen; p. 217-230.

23. Feijó CM, Spaine DM, Tibaldi DS, et al. Atlas de Reprodução Humana. In: Dzik A, Donadio NF, Esteves SC, Nagy ZP. Segmento Farma; 2012. Capítulo 4, Espermograma; p. 45-54.

24. WHO - World Health Organization. WHO laboratory manual for the examination and processing of human sêmen. 5. Ed. Switzerland, 2009.

25. Cooper TG, Noonan E, Eckardstein SV, et al. World Health Organization reference values for human sêmen characteristics. Human Reproduction Update. 2010 Nov; 16(3): 231-245, doi:10.1093/humupd/dmp048.

26. Pasqualotto EB, Ferreira RV, Fonseca GP, et al. A análise seminal deve ser requisitada para homens com histórico de fertilidade prévia? Rev Bras Ginecol Obstet. 2006 Nov; 28(11): 652-657, dx.doi.org/10.1590/S0100-72032006001100004.

27. Andrade-Rocha FT. O papel do exame do sêmen na infertilidade masculina. IV- Avaliação da interação espermatozoide-muco cervical. RBAC. 2002; 34(1): 31-37.

28. Mclachlan RI, Yazdani A, Kovacs G. et al. Management of the Infertile Couple. Aust Fam Physician. 2005; 34(3): 111-117. PMID: 15799655.

29. Santos RC. Estudo relaciona alcoolismo a lesões na prostata. Jornal da UNICAMP. 2003 Out; 2: 4, http://www.unicamp.br/unicamp/unicamp_hoje/ju/outubro2003/ju232pag4b.html.

30. Esteves SC. Male infertility due to spermatogenic failure: current management and future perspectives. Anim. Reprod. 2015 Jan/Mar; 12(1): 62-80.

31. Esteves SC, Miyaoka R, Agarwal A. An update on the clinical assessment of the infertile male. CLINICS. 2011; 66(4): 691-700, doi:10.1590/s1807-59322011000400026.

32. Aghamohammadi A, Zafari M. The impact of cigarette smoking on sperm parameters: A crosssectional study. International Conference on Environmental, Biomedical and Biotechnology. IPCBEE. 2011; 16 : 81-84, http://www.ipcbee.com/vol16/17-E20004.pdf.

33. Ahmadnia H, Ghanbari M, Moradi MR, et al. Effect of cigarette smoke on spermatogenesis in rats. J Urol. 2007; 4(4): 159-163. PMID: 17987579.

34. Heidary F, Ahmadi R, Lotfi A. The Effects of Cigarette or Hookah Smoking on Serum Levels of LH, FSH or Testosterone in Male Rats. Bangkok, International Conference on Medical, Biological and $\begin{array}{lllll}\text { Pharmaceutical } & \text { Sciences. } & \text { ICMBPS. } & & \\ \end{array}$ http://psrcentre.org/images/extraimages/30.\%20312074.pdf.

35. Andrade-Roha FT. O papel do exame do sêmen na infertilidade masculina. III- Testes para função espermática. RBAC. 1998; 30: 13-21.

36. ASRM - American Society for Reproductive Medicine. Diagnostic Evaluation of the infertile male: a committee opinion. Fertility and sterility. 2015 Mar; 103(3): 18-25, doi:10.1016/j.fertnstert.2014.12.103. 